\title{
HIGHEST WEIGHTS FOR CATEGORICAL REPRESENTATIONS
}

\author{
DAVID BEN-ZVI, SAM GUNNINGHAM, AND HENDRIK OREM
}

\begin{abstract}
We present a criterion for establishing Morita equivalence of monoidal categories, and apply it to the categorical representation theory of reductive groups $G$. We show that the "de Rham group algebra" $\mathcal{D}(G)$ (the monoidal category of $\mathcal{D}$-modules on $G$ ) is Morita equivalent to the universal Hecke category $\mathcal{D}(N \backslash G / N)$ and to its monodromic variant $\widetilde{\mathcal{D}}(B \backslash G / B)$. In other words, de Rham $G$-categories, i.e., module categories for $\mathcal{D}(G)$, satisfy a "highest weight theorem" - they all appear in the decomposition of the universal principal series representation $\mathcal{D}(G / N)$ or in twisted $\mathcal{D}$-modules on the flag variety $\widetilde{\mathcal{D}}(G / B)$.
\end{abstract}

\section{INTRODUCTION}

1.1. Morita equivalences. The fundamental building block for representations of reductive groups is the principal series, the collection of representations constructed by parabolic induction from a maximal torus. These representations can be described as the constituents of the appropriate space of functions on the coset space $G / N$ (where $N$ is the unipotent radical of a Borel subgroup $B$ ), and are parametrized by modules for the Hecke algebra of double cosets $N \backslash G / N$. In the setting of finite dimensional representations of compact or complex reductive groups, the Borel-Weil theorem asserts that all representations are principal series so that we obtain a complete classification of representations based on their highest weights, as representations of the torus $H=B / N$, and their symmetries, identified with the Weyl group. The Casselman subrepresentation theorem provides an analogous picture for real reductive groups, showing that any irreducible admissible representation arises as a subrepresentation of a principal series representation. This assertion fails if we keep track of unitary structure, for example due to existence of discrete series. Likewise for representations of finite and p-adic reductive groups, analogous statements fail due to the existence of cuspidal representations. Our goal in this paper is to prove the corresponding result for the categorical representations of a complex reductive group $G$ : they all appear in the principal series, so that there are no cuspidal categorical representations.

Just as representations of a group $G$ arise naturally as spaces of functions on $G$-spaces, categorical representations of an algebraic group $G$ arise naturally as categories of sheaves on $G$-varieties $X$. Such categories are automatically module categories for the corresponding "group algebra", the monoidal category of sheaves on $G$ equipped with the convolution product. (In order to perform "functional analytic" operations on categories of sheaves it is essential to live in an appropriate setting of homotopical algebra, which for us will be the $\infty$-category of differential graded categories.)

Two natural classes of sheaves to consider are quasi coherent sheaves and algebraic $\mathcal{D}$-modules. Throughout, given a stack $X$, we denote by $Q C(X)$ and $\mathcal{D}(X)$ the $\infty$-categories of quasi coherent sheaves and $\mathcal{D}$-modules on $X$ GR. Thus we arrive at the notions of algebraic (or weak) and de Rham (or strong) categorical representations of $G$. These are module categories for the "algebraic group algebra" $Q C(G)$ or the "de Rham group algebra" $\mathcal{D}(G)$. The former (weak) notion is simpler - in particular the definitive work of Gaitsgory [G2] shows algebraic $G$-categories are generated by 
the trivial module, Vect $=Q C(p t)$. The latter (strong) notion, whose study was pioneered in $[\mathrm{BD}$, is the most relevant to geometric representation theory. In particular the adjoint action of $G$ on $\mathfrak{g}$ endows the category $\mathcal{U}(\mathfrak{g})-\bmod$ with a de Rham $G$-action; it was shown by Beraldo (using Gaitsgory's 1-affineness result) that de Rham $G$-categories are generated by the category of $\mathcal{U}(\mathfrak{g})$ modules. Beilinson-Bernstein localization can be seen as a relation between this representation and the geometric representation given by twisted $\mathcal{D}$-modules on the flag variety, or equivalently the "monodromic principal series" category $\mathcal{D}(G / N)_{H}$ of $H$-monodromic (i.e., weakly $H$-equivariant) $\mathcal{D}$-modules on $G / N$.

To explain the utility of having generators for the category of $G$-categories, recall the following fundamental result of Morita theory: if an abelian category $\mathcal{C}$ has a compact projective generator $M$, then $\mathcal{C}$ is equivalent to the category of modules for the corresponding Hecke algebra $\mathcal{H}(M):=$ $\operatorname{End}_{\mathcal{C}}(M)$. The theorem of Gaitsgory asserts that the trivial algebraic $G$-category, Vect behaves as if it were a projective generator of the category of algebraic $G$-categories; in particular, the category of algebraic $G$-categories is equivalent to modules for the monoidal category $\operatorname{Rep}(G)=$ $\operatorname{End}_{Q C(G)}($ Vect $)$. This situation is as nice as possible: the monoidal category $\operatorname{Rep}(G)$ is rigid, symmetric, and semisimple, making it amenable to study by algebraic and combinatorial methods. Similarly, Beraldo's result asserts that de Rham $G$-categories are equivalent to module categories for the monoidal category $H C_{G}=\operatorname{End}_{\mathcal{D}(G)}(\mathcal{U}(\mathfrak{g})-\bmod )$ of Harish-Chandra bimodules. The monoidal category $H C_{G}$ is rigid (although it is not symmetric and it is far from being semisimple).

Our main result asserts that all de Rham $G$-categories are generated by either the principal series category $\mathcal{D}(G / N)$ or its monodromic version $\mathcal{D}(G / N)_{H}$.

Theorem 1.1. Let $G$ be a complex reductive group, with Borel $B, N=[B, B]$, and $H=B / N$. The $\mathcal{D}(G)-\mathcal{D}(N \backslash G / N)$-bimodule $\mathcal{D}(G / N)$ defines a Morita equivalence between the monoidal categories $\mathcal{D}(G)$ and $\mathcal{D}(N \backslash G / N)$.

Corollary 1.2. The following monoidal categories are all Morita equivalent.

(1) $\mathcal{D}(G)$,

(2) $\mathcal{D}(N \backslash G / N)$,

(3) $\mathcal{D}_{H}(N \backslash G / N)_{H}$,

(4) $H C_{G}$.

To prove Theorem 1.1] we will formulate a more general statement giving sufficient conditions for when a module category $M$ for a monoidal category $A$ defines a Morita equivalence between $A$ and $\mathcal{H}(M):=\operatorname{End}_{A}(M)$.

For the purposes of analogy, let us consider the following situation. Let $A$ be a finite dimensional algebra over a field $k$, and $M$ a right $A$-module. Note that $M$ is naturally a $\mathcal{H}(M)-A$-bimodule, so determines a functor

$$
M \otimes_{A}(-): A-\bmod \rightarrow \mathcal{H}(M)-\bmod
$$

Now suppose that $M$ is projective and faithful as an $A$-module. In that case the module $M$ defines a Morita equivalence between $A$ and $\mathcal{H}(M)$, which is to say, the functor $M \otimes_{A}(-)$ defines an equivalence between $A-\bmod$ and $\mathcal{H}(M)-\bmod$.

Now let $A$ and $B$ be monoidal $\infty$-categories, and $M={ }_{B} M_{A}$ a bimodule category. As above, we think of $M$ as defining a functor:

$$
M \otimes_{A}(-): A-\bmod \rightarrow B-\bmod .
$$

We say that $M$ has a left (respectively right) dual if the corresponding functor has a left (respectively right) adjoint. A bimodule $M$ is proper dualizable if $M$ is dualizable as an $A$-module, and the 
evaluation and coevaluation maps admit continuous right adjoints (see Definition 2.7 for the full definition).

Our key technical result is the following

Theorem 1.3. Suppose $M={ }_{B} M_{A}$ is a proper dualizable bimodule. Then there is a fully faithful embedding

$$
i: \mathcal{H}(M)-\bmod \hookrightarrow A-\bmod .
$$

If, in addition, the action map $A \rightarrow \operatorname{End}_{B}(M)$ is conservative then $i$ is an equivalence of categories.

We will apply this result in the case $A=\mathcal{D}(G), B=\mathcal{D}(H)$, and $M=\mathcal{D}(N \backslash G)$ to obtain the equivalence between $\mathcal{D}(G)-\bmod$ and $\mathcal{D}(N \backslash G / N)-\bmod$ in Theorem 1.1

1.2. Acknowledgments. We would like to acknowledge the National Science Foundation for its support through individual grant DMS-1103525 (DBZ).

\section{Abstract Morita Theory}

2.1. $\infty$-categorical preliminaries. Throughout, we rely on the foundations developed in L2]. Let $k$ be a field, and let DGCat denote the $(\infty, 1)$ category of $k$-linear, stable, presentable $\infty$-categories where morphisms are functors which are left adjoints (equivalently, functors which preserve small colimits). Recall that DGCat comes equipped with a symmetric monoidal structure $\otimes$. The unit object of DGCat is the category $S=$ Vect $_{k}$ of (differential graded) $k$-vector spaces.

Recall that a pair of adjoint functors:

$$
G: C \leftrightarrows D: F
$$

(where $G$ is left adjoint to $F$ ) give rise to a monad $F G$ acting on $C$. We will denote by $C^{F G}$ the category of modules for $F G$ in $C$.

Theorem 2.1 (Barr-Beck-Lurie, easy version [L2, Corollary 4.7.4.16). Let $F: D \rightarrow C$ be a morphism in DGCat (in particular, $F$ preserves colimits) which admits a left adjoint $G$. Then $F$ factors canonically as

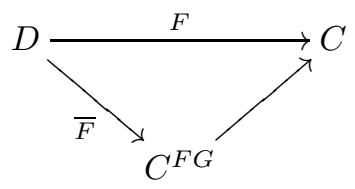

where $\bar{F}$ has a fully faithful left adjoint. If, in addition, $F$ is conservative then $\bar{F}$ is an equivalence (in that case, we say that $F$ is monadic).

The following lemma states that certain colimits in DGCat can be computed as limits of the corresponding diagram of right adjoints. The result appears as Lemma 1.3.3 in G1]; it is a consequence of Corollary 5.3.3.4 in L1].

Lemma 2.2. Let $I$ be an $\infty$-category, and $I \rightarrow$ DGCat, $i \mapsto C_{i}$ a functor. For each $\alpha: i \rightarrow j$, let $F_{\alpha}: C_{i} \rightarrow C_{j}$ denote the corresponding functor. Suppose each $F_{\alpha}$ admits a continuous right adjoint, and consider the corresponding diagram $I^{o p} \rightarrow$ DGCat formed by taking the right adjoints of each $F_{\alpha}$. Then there is a canonical equivalence

$$
\operatorname{colim}_{i \in I}\left(C_{i}\right) \stackrel{\sim}{\longrightarrow} \varliminf_{j \in I^{o p}}\left(C_{j}\right) .
$$


The theory of monadic descent in the context of $\infty$-categories was developed by Lurie in [L2], Chapter 4.7.6. The following definition and theorem are what we require in this paper.

Definition 2.3. Let $C^{\bullet}$ be a cosimplicial object (respectively, augmented cosimplicial object) of DGCat; we say that $C^{\bullet}$ satisfies the monadic Beck-Chevalley conditions, if the following two conditions hold:

(1) For every object $[i]$ in the simplex category $\Delta$ (respectively the augmented simplex category $\left.\Delta^{+}\right)$, the last face map $C^{\partial_{i}^{0}}: C^{i} \rightarrow C^{i+1}$ admits a left adjoint $\left(C^{\partial_{i}^{0}}\right)^{L}$;

(2) For every morphism $\alpha:[i] \rightarrow[j]$ in $\Delta$ (respectively $\Delta^{+}$), the diagram

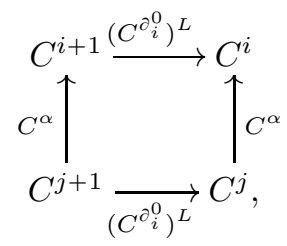

commutes.

Theorem 2.4 (Monadic descent, [L2, Theorem 4.7.6.2, Corollary 4.7.6.3). (1) Let $\widetilde{C} \bullet$ be an coaugmented cosimplicial object of DGCat which satisfies the monadic Beck-Chevalley conditions. Let $C^{\bullet}$ denote the cosimplical object without the coaugmentation. Then the canonical map $\bar{F}: \widetilde{C}^{-1} \rightarrow \operatorname{Tot}\left(C^{\bullet}\right)$ admits a fully faithful right adjoint. If the coaugmentation $\widetilde{C}^{-1} \rightarrow C^{0}$ is conservative, then $\bar{F}$ is an equivalence.

(2) Let $C^{\bullet}$ be a cosimplicial object of DGCat. Then the coaugmented diagram $\operatorname{Tot}\left(C^{\bullet}\right) \rightarrow C^{\bullet}$ satisfies the monadic Beck-Chevalley conditions.

2.2. Monoidal categories, modules, and dualizibility. In this paper, a monoidal category means an algebra object in DGCat, in the sense of [L2], Chapter 4. Given a monoidal category $A$, we have the notion of a (left or right) module category $M$ for $A$ ( [L2], Chapter 4.2, 4.3). In particular this means that $M$ and the structure maps $A \otimes M \rightarrow M$ are in DGCat. We write $A$ - mod for the category of left $A$-module objects in DGCat, and we identify the category of right $A$-modules with $A^{o p}$ - mod (note that $A^{o p}$ here refers to $A$ with the opposite monoidal structure, not the opposite category). Given monoidal categories $A$ and $B$, a $B-A$-bimodule is the same thing as an object of $B \otimes A^{o p}-\bmod$.

Given an $A-A$-bimodule $M$, the Hochshild homology category $H H(A ; M)$ can be identified with the colimit of the simplicial bar complex $\operatorname{Bar} .(A ; M)$, whose $n$-simplices are given by $M \otimes A^{\otimes n}$. In the case where the bimodule $M$ is of the form $L \otimes N$, where $L$ is a left $A$-module, and $N$ is a right $A$-module, the Hochshild homology $H H(A ; L \otimes N)$ can be identified with the relative tensor product $L \otimes_{A} N$. In that case we will often write $\operatorname{Bar} .(A ; L, N)$ for the corresponding bar complex.

Suppose $A$ and $B$ are monoidal categories, and $M$ a $B-A$-bimodule. Thus $M$ determines a functor

$$
M \otimes_{A}(-): A-\bmod \rightarrow B-\bmod .
$$

Definition 2.5. We say that $M={ }_{B} M_{A}$ is $A$-dualizable (or left dualizable) if $M_{A} \otimes_{A}(-$ ) has a left adjoint. Similarly, $M$ is $B$-dualizable (or right dualizable) if $M \otimes_{A}(-)$ has a right adjoint. 
More explicitly, the bimodule $M$ is $A$-dualizable if and only if there exists an $A-B$-bimodule ${ }^{\vee} M$, together with morphisms

$$
\begin{aligned}
& u^{L}: B \rightarrow M \otimes_{A}{ }^{\vee} M, \\
& c^{L}:{ }^{\vee} M \otimes_{B} M \rightarrow A,
\end{aligned}
$$

such that the triangle identities hold, that is, the composite functors

$$
\begin{array}{r}
M \stackrel{u_{L} \otimes 1_{M}}{\longrightarrow} M \otimes_{A}{ }^{\vee} M \otimes_{B} M \stackrel{1_{M} \otimes c_{L}}{\longrightarrow} M \\
{ }^{\vee} M \stackrel{1 \vee_{M} \otimes u^{L}}{\longrightarrow}{ }^{\vee} M \otimes_{B} M \otimes_{A}{ }^{\vee} M \stackrel{c^{L} \otimes 1 \vee_{M}}{\longrightarrow} \vee M
\end{array}
$$

are equivalences.

Similarly, $M$ is $B$-dualizable if there exists an $A-B$-bimodule $M^{\vee}$, together with morphisms:

$$
\begin{aligned}
& c^{R}: M \otimes_{A} M^{\vee} \rightarrow B, \\
& u^{R}: A \rightarrow M^{\vee} \otimes_{B} M,
\end{aligned}
$$

such that the corresponding triangle identities hold.

Remark 2.6. (1) Note that the condition that $M$ be $A$ dualizable (repectively $B$ ) depends only on the structure of $M$ as an $A$-module (respectively, a $B$-module). To see this, note that the data $\left(u^{L}, c^{L}\right)$ is equivalent to data:

$$
\begin{aligned}
& u_{0}^{L}: S \rightarrow M \otimes_{A}{ }^{\vee} M, \\
& c^{L}:{ }^{\vee} M \otimes_{S} M \rightarrow A,
\end{aligned}
$$

satisfying the triangle condition (recall that $S=$ Vect $_{k}$ denotes the unit of DGCat). In other words, the bimodule ${ }_{B} M_{A}$ being left dualizable is equivalent to ${ }_{S} M_{A}$ being left dualizable.

(2) If $M$ is left dualizable, then for any right $A$-module $N$, we have

$$
\operatorname{Hom}_{A^{o p}}(M, N) \simeq N \otimes_{A}{ }^{\vee} M .
$$

(Here $A^{o p}$ refers to $A$ with the opposite monoidal structure).

(3) If $M$ is left dualizable, we have an identification

$$
{ }^{\vee} M \otimes_{A} M \simeq \operatorname{Hom}_{A}(M, M) .
$$

The unit morphism $u^{L}$ becomes identified with the inclusion of the unit object in the monoidal category $S \rightarrow \mathcal{H}(M)=\operatorname{Hom}_{A}(M, M)$.

(4) A sufficient condition for $M$ to be dualizable as an $S$-module is that it is compactly generated. In that case $M^{\vee}=\operatorname{Ind}\left(M_{c}^{\diamond}\right)$, where $M_{c}^{\diamond}$ means the opposite category of the subcategory of compact objects.

Definition 2.7. We say that the $B-A$-bimodule $M={ }_{B} M_{A}$ is properly dualizable if $M$ is $A$ dualizable and the evaluation and coevaluation morphisms have continuous right adjoints.

Remark 2.8. Suppose $F: A \rightarrow B$ is a morphism in DGCat where $A, B$ are compactly generated. Then $F$ has a continuous right adjoint if and only if $F$ sends compact objects to compact objects.

Lemma 2.9. Suppose $M={ }_{B} M_{A}$ is a properly dualizable bimodule. Then $M$ is B-dualizable. Moreover, the B-dual $M^{\vee}$ is equivalent to the A-dual ${ }^{\vee} M$, and the unit (respectively counit) morphism for the right duality is given by the right adjoint of the counit (respectively unit) for the left duality. 
Proof. As $M$ is left dualizable, we have functors:

$$
\begin{aligned}
& u^{L}: B \rightarrow M \otimes_{A}{ }^{\vee} M, \\
& c^{L}:{ }^{\vee} M \otimes_{B} M \rightarrow A,
\end{aligned}
$$

such that the triangle identities of Equation 3 are satisfied. Taking right adjoints, we obtain functors:

$$
\begin{aligned}
& c^{R}: M \otimes_{A}{ }^{\vee} M \rightarrow B, \\
& u^{R}: A \rightarrow{ }^{\vee} M \otimes_{B} M,
\end{aligned}
$$

where $u^{R}$ is the right adjoint of $c^{L}$ and $c^{R}$ is the right adjoint of $u^{L}$. By the assumption of proper dualizability, $u^{R}$ and $c^{R}$ are continuous, i.e., morphisms in DGCat. Moreover, we observe that the functors $\left(c^{R}, u^{R}\right)$ satisfy the triangle identities for ${ }^{\vee} M$ to be the right dual to $M$, by taking the right adjoints of the triangle identites for the left duality $\left(c^{L}, u^{L}\right)$.

2.3. Rigid monoidal categories. A monoidal category $A$ is called rigid if it is compactly generated, and every compact object is (left and right) dualizable 1 Given a rigid monoidal category $A$, let $A^{\prime}$ denote its dual as an object of DGCat (recall that this can be constructed as the Indcategory of the opposite category of compact objects of $A$ ). The operations taking left and right duals define functors

$$
\phi^{R}, \phi^{L}: A \rightarrow A^{\vee}
$$

The composition $\varphi:\left(\phi^{R}\right)^{-1} \phi^{L}$ is a monoidal autoequivalence of $A$. A pivotal structure on $A$ is a monoidal equivalence of functors $\alpha: \varphi \simeq i d_{A}$.

Theorem 2.10 (Gaitsgory, G2] Appendix D). Let A be a rigid monoidal category.

(1) A left $A$-module $L$ is dualizable if and only if $L$ is dualizable as an object of DGCat.

(2) If $A$ is equipped with a pivotal structure and $M$ is an A-bimodule, there is a canonical identification

$$
H H_{*}(A ; M) \simeq H H^{*}(A ; M) .
$$

Remark 2.11. Part 2 of Theorem 2.10 is proved by observing that the structure morphisms in the simplicial bar object computing $H H_{*}(A ; M)$ have continuous right adjoints, and the corresponding diagram of right adjoints is identified with the cosimplicial cobar object computing $H H^{*}(A ; M)$. The identification of $H H_{*}(A ; M)$ and $H H^{*}(A ; M)$ then follows from Lemma 2.2

\section{Proof of Theorem 1.3}

Fix monoidal categories $A$ and $B$, and properly dualizable bimodule $M={ }_{B} M_{A}$ with dual ${ }^{\vee} M=\operatorname{Hom}_{A^{o p}}(M, A)$.

Note that the Hecke algebra $\mathcal{H}:=\mathcal{H}(M):=\operatorname{End}_{A}(M)$ is identified with $M \otimes_{A} \vee M$, by $A$-duality. There is an algebra morphism $B \rightarrow \mathcal{H}$ given by the action of $B$ on $M$. Thus we may consider $\mathcal{H}$ as an algebra object in $B-B$-bimodules. The $B-A$-bimodule $M$ carries a canonical left action of $\mathcal{H}(M)$ which commutes with the $A$-action, thus promoting $M$ to a $\mathcal{H}(M)-A$-bimodule; similarly, ${ }^{\vee} M$ is naturally a $A-\mathcal{H}(M)$ bimodule.

\footnotetext{
${ }^{1}$ There is a more general definition of rigid monoidal category which does not require compact generation, see G2, D.1.1. The definitions agree in the compactly generated case.
} 
To compute the relative tensor product ${ }^{\vee} M \otimes_{\mathcal{H}} M$, we will use the bar construction of $\mathcal{H}$ considered as an algebra object in the monoidal category of $B-B$-bimodules. Explicitly, we define the simplicial category $C$ • with simplices

$$
\begin{aligned}
C_{n} & ={ }^{\vee} M \otimes_{B} \mathcal{H}^{\otimes_{B} n} \otimes_{B} M \\
& \simeq{ }^{\vee} M \otimes_{B}\left(M \otimes_{A}{ }^{\vee} M\right)^{\otimes_{B} n} \otimes_{B} M \\
& \simeq\left({ }^{\vee} M \otimes_{B} M\right)^{\otimes_{A}(n+1)} .
\end{aligned}
$$

The face maps $C_{\partial_{n}^{i}}: C_{n} \rightarrow C_{n-1}$ are given by inserting $c^{L}:{ }^{\vee} M \otimes_{B} M \rightarrow A$ in the $i$ th tensor factor of

$$
C_{n} \simeq\left({ }^{\vee} M \otimes_{B} M\right)^{\otimes_{A}(n+1)},
$$

and the degeneracy maps $C_{e_{n}^{j}}: C_{n} \rightarrow C_{n+1}$ are given by inserting $u^{L}: B \rightarrow M \otimes_{B}{ }^{\vee} M$ after the $j$ th tensor factor of

$$
C_{n} \simeq{ }^{\vee} M \otimes_{B}\left(M \otimes_{A}{ }^{\vee} M\right)^{\otimes_{B} n} \otimes_{B} M
$$

If we set $C_{-1}=A$, the morphism $c^{L}: C_{0} \rightarrow C_{-1}$ defines an augmentation of $C_{\bullet}$ defining an augmented simplicial object $\widetilde{C}_{\bullet}$. As the functors $c^{L}$ and $u^{L}$ have continuous right adjoints, it follows that the structure maps of $C$. have continuous right adjoints, and thus the cosimplicial category $C^{\bullet}$ obtained by taking right adjoints is a cosimplicial object in DGCat.

Lemma 3.1. The coaugmented cosimplicial category $\widetilde{C}^{\bullet}$ satisfies the monadic Beck-Chevalley conditions.

Proof. We should check that, for each $\alpha:[i] \rightarrow[j]$ in $\Delta^{+}$, the square

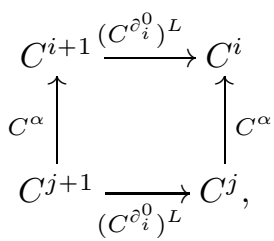

commutes. For example, in the case when $\alpha$ is the unique map $[-1] \rightarrow[0]$, we have the diagram

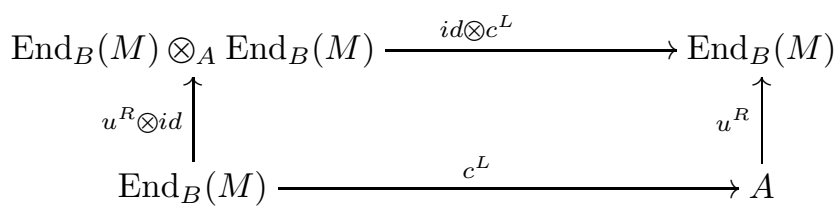

which commutes by inspection. The remaining cases are similar.

Note that we have a commutative diagram of monoidal functors:

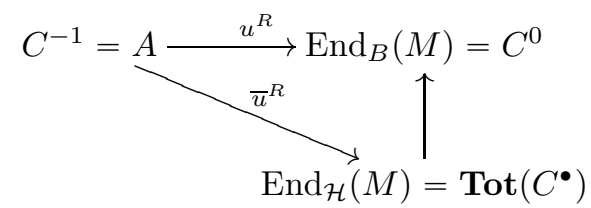


Proposition 3.2. The functor $\overline{u^{R}}$ has a fully faithful, continuous, monoidal left adjoint, $\bar{c}^{L}$, and the essential image of $\bar{c}^{L}$ is Morita equivalent to $\mathcal{H}$. Moreover, if $F$ is conservative, then $\bar{F}$ is an equivalence.

Proof. The augmented cosimplicial object $\widetilde{C}^{\bullet}$ satisfies the Beck-Chevalley conditions. Thus, by Theorem 2.4, $\bar{u}^{R}$ has a fully faithful left adjoint $\bar{c}^{L}$. Moreover, if $u^{R}$ is conservative, then $\bar{u}^{R}$ is an equivalence.

The functor $\bar{c}^{L}$ is naturally monoidal, by virtue of being the fully faithful left adjoint to the monoidal map $\bar{u}^{R}\left(\bar{c}^{L}\right.$ is naturally oplax monoidal as the left adjoint to $\bar{u}^{R}$; as $\bar{u}^{R} \bar{c}^{L} \simeq i d_{\operatorname{End}_{B}(M)}$, it follows that the monoidal structure of $\bar{c}^{L}$ is strong).

It remains to show that $M$ defines a Morita equivalence between the essential image of $\bar{c}^{L}$ and $\mathcal{H}$. Replacing $A$ by the essential image of $\bar{c}^{L}$, we may assume $u^{R}$ is conservative. Note that

$$
\begin{aligned}
{ }^{\vee} M \otimes_{\mathcal{H}} M & \simeq|C \cdot| \\
& \simeq \operatorname{Tot}\left(C^{\bullet}\right) \\
& \simeq A .
\end{aligned}
$$

Note also that the augmented cosimplicial category $\widetilde{C} \bullet$ is naturally an augmented cosimplicial object of $A-A$-bimodules. In particular, the morphism $\bar{u}^{R}$ is a morphism of $A-A$-bimodules. As we have already noted $\mathcal{H} \simeq M \otimes_{A}{ }^{\vee} M$ as $\mathcal{H}-\mathcal{H}$-bimodules, and thus $A$ is Morita equivalent to $\mathcal{H}$ as claimed.

Remark 3.3. The Beck-Chevalley conditions for the augmented cosimplicial object $\widetilde{C}^{\bullet}$ imply a projection formula for the adjunction $u^{R}: A \leftrightarrows \operatorname{End}_{B}(M): c^{L}$

$$
c^{L}\left(u^{R}(a) * b\right) \simeq a * c^{L}(b) .
$$

In particular, the object $\operatorname{Spr}_{M}:=c^{L}\left(i d_{M}\right) \in A$ is a coalgebra object which we call the Springer object. 2 The comonad $c^{L} u^{R}$ acting on $A$ is given by $a \mapsto S p r_{M} * a$. Similarly, the object $e_{M}:=\bar{c}^{L}\left(i d_{M}\right)$ is an idempotent coalgebra object (it is the unit for the monoidal structure on the essential image of $\overline{c^{L}}$ ), which represents the idemotent comonad $\bar{c}^{L} \bar{u}^{R}$. In other words, the essential image of $\bar{c}^{L}$ is naturally identified with $e_{M}$-comodule objects in $A$.

\section{Modules for the CAtegorical group algebra}

4.1. $G$-invariants and coinvariants. In order to apply the results of the previous section, we need the following important consequence of Gaitsgory's 1-affineness theorem.

Theorem 4.1 ( $\mathrm{G} 2$, Be Theorem 3.5.7). For any complex reductive group $G$, the $\mathcal{D}(G)$-module $\mathcal{U}(\mathfrak{g})-\bmod$ defines a Morita equivalence between $\mathcal{D}(G)$ and $\operatorname{End}_{\mathcal{D}(G)}(\mathcal{U}(\mathfrak{g})) \simeq H C_{G}$.

Remark 4.2. The functor $(-) \otimes_{\mathcal{D}(G)}(\mathcal{U}(\mathfrak{g})-\bmod )$ from $\mathcal{D}(G)-\bmod$ to $H C_{G}-\bmod$ is known as the the functor of weak $G$-coinvariants. Given a stack $X$ with an action of $G, \mathcal{D}(X)$ is a $\mathcal{D}(G)$ module, and the corresponding $H C_{G}$-module, $\mathcal{D}(X) \otimes_{\mathcal{D}(G)}(\mathcal{U}(\mathfrak{g})$ - mod), can be identifed with the category $\mathcal{D}(G \backslash X)$ of $G$-weakly equivariant objects of $\mathcal{D}(X)$.

Proposition 4.3 ( $[\mathrm{Be}]$, section 2.5). The monoidal category $H C_{G}$ is rigid and pivotal.

\footnotetext{
${ }^{2}$ In the case $A=\mathcal{D}(G), M=\mathcal{D}(G / N)$, and $B=\mathcal{D}(H)$ (using the notiation of Section 3), the object $\operatorname{Spr}_{M}$ is the Springer sheaf.
} 
Proposition 4.3 follows from the fact that there is a monoidal functor $\operatorname{Rep}(G) \rightarrow H C_{G}$ with a continuous and conservative right adjoint, and the fact that $\operatorname{Rep}(G)$ itself is rigid and pivotal.

Given a $\mathcal{D}(G)$-module $M$, let $M^{G}=\operatorname{Hom}_{\mathcal{D}(G)}($ Vect, $M)$ denote the strong $G$-invariants, and $M_{G}=\operatorname{Vect} \otimes_{\mathcal{D}(G)} M$ the strong $G$-coninvariants.

Proposition 4.4 ( $\left[\mathrm{Be}\right.$, Theorem 3.6.1). If $M$ is a $\mathcal{D}(G)$-module, then $M_{G} \simeq M^{G}$.

Now let $X$ be a perfect stack with an action of $G$. We have the Cech simplicial stack with $n$-simplices $X \times G^{\times n}$ which is augmented by $X / G$. Taking categories of $D$-modules, we get a cosimplicial category with $n$-simplices $\mathcal{D}\left(X \times G^{\times n}\right)$, and structure maps of the form $f^{!}$for each map $f$ in the Cech simplicial stack. After identifying $\mathcal{D}\left(X \times G^{\times n}\right)$ with $\mathcal{D}(X) \otimes \mathcal{D}(G)^{\otimes n}$, this cosimplicial category becomes identified with $\operatorname{Bar}^{\bullet}(\mathcal{D}(G) ; \mathcal{D}(X)$, Vect). Thus, there is a natural equivalence $\mathcal{D}(X)^{G} \simeq \mathcal{D}(X / G)$. The result of Beraldo above implies that we can also identify the coinvariants $\mathcal{D}(X)_{G}$ with $\mathcal{D}(X / G)$. In fact, we have the following result.

Proposition 4.5 ( $\mathrm{Be}]$ ). Let $X$ be a perfect stack with an action of $G$. The canonical augmentations

$$
\mathcal{D}(X / G) \stackrel{p^{!}}{\longrightarrow} \operatorname{Bar}^{\bullet}(\mathcal{D}(G) ; \mathcal{D}(X), \text { Vect })
$$

and

$$
\operatorname{Bar}_{\bullet}(\mathcal{D}(G) ; \mathcal{D}(X), \text { Vect }) \stackrel{p_{*}}{\longrightarrow} \mathcal{D}(X / G)
$$

realize $\mathcal{D}(X / G)$ as the totalization and geometric realization respectively.

Corollary 4.6. Let $G$ be a reductive groups and $X$ and $Y$ perfect stacks with an action of $G$. Then there is a canonical equivalence

$$
\mathcal{D}(X) \otimes_{\mathcal{D}(G)} \mathcal{D}(Y) \simeq \mathcal{D}((X \times Y) / G) .
$$

Lemma 4.7. Let $G$ and $H$ be reductive groups and $X$ a perfect stack with an action of $G \times H$. Then $\mathcal{D}(X)$ is $\mathcal{D}(G)$ and $\mathcal{D}(H)$ dualizable and self dual as a $\mathcal{D}(H)-\mathcal{D}(G)$-bimodule. Moreover, after identifying $\mathcal{D}(X) \otimes_{\mathcal{D}(H)} \mathcal{D}(X)$ with $\mathcal{D}(H \backslash(X \times X))$ and $\mathcal{D}(X) \otimes_{\mathcal{D}(G)} \mathcal{D}(X)$ with $\mathcal{D}((X \times X) / G)$ using Corollary 4.6 , the duality data

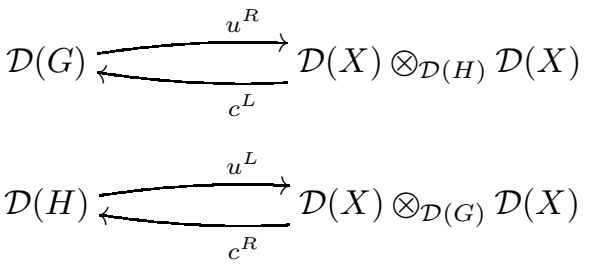

are given by:

$$
\begin{aligned}
u^{L} & =\delta_{*} \gamma^{!} \\
c^{L} & =\alpha_{*} \beta^{!} \\
u^{R} & =\beta_{*} \alpha^{!} \\
c^{R} & =\gamma_{*} \delta^{!},
\end{aligned}
$$


where $\alpha, \beta, \gamma, \delta$ are the canonical maps in the following correspondences:

$$
\begin{aligned}
& G \stackrel{\alpha}{\longleftarrow}(H \backslash X) \times G \stackrel{\beta}{\longrightarrow} H \backslash(X \times X) \\
& H \longleftarrow{ }^{\longleftarrow} H \times(X / G) \stackrel{\delta}{\longrightarrow}(X \times X) / G .
\end{aligned}
$$

Proof. Suppose one takes (as an ansatz) the unit and counit maps to be as claimed above; it then follows from base change that the triangle identities are satisfied, and thus the ansatz unit and counit are indeed the unit and counit of an adjunction as required.

4.2. Categorical Highest Weight Theorem. Fix a reductive group $G$, a Borel subgroup $B$, and take $N=[B, B], H=B / N$, and $X=N \backslash G$.

Proposition 4.8. The $\mathcal{D}(H)-\mathcal{D}(G)$-bimodule $\mathcal{D}(N \backslash G)$ is properly dualizable and self dual, and the action morphism $u^{R}$ is conservative.

Proof. The dualizability follows from Lemma 4.7 We have $u^{L}=\delta_{*} \gamma^{!}$, and $c^{L}=\alpha_{*} \beta^{!}$(where $\alpha, \beta, \gamma, \delta$ are as in Diagram (13). In this case, $\alpha$ and $\delta$ are proper, and $\beta$ and $\gamma$ are smooth, thus $\alpha_{*}$, $\delta_{*}, \beta^{!}$, and $\gamma^{!}$all have continuous right adjoints as required.

It remains to show that $u^{R}$ is conservative. As observed by Mirkovic-Vilonen [MV1, the composite $c^{L} u^{R}$ is the endofunctor on $\mathcal{D}(G)$ given by convolution with the Springer sheaf $\mathcal{S}_{G}:=(\widetilde{\mathcal{N}} \rightarrow$ $\mathcal{N})_{*} \omega_{\widetilde{\mathcal{N}}}$. As the Springer sheaf contains the unit object for convolution $\delta_{e} \in \mathcal{D}(G)$ as a direct summand, we have that $c^{L} u^{R}$ contains the indentity functor as a summand. In particular, $u^{R}$ is conservative.

Corollary 4.9. The bimodule $\mathcal{D}(N \backslash G)$ defines a Morita equivalence between $\mathcal{D}(G)$ and $\mathcal{H}=$ $\mathcal{D}(N \backslash G / N)$.

Theorem 4.10. The bimodule $\mathcal{D}(N \backslash G / N)_{H}$ defines a Morita equivalence between $\mathcal{D}(N \backslash G / N)$ and $\mathcal{D}_{H}(N \backslash G / N)_{H}$.

Proof. The functor

$$
M \mapsto M \otimes_{\mathcal{D}(N \backslash G / N)} \mathcal{D}(N \backslash G / N)_{H}
$$

the functor of weak $H$-invariants. Thus the result follows from the 1-affineness theorem of Gaitsgory G2.

\section{REFERENCES}

[BD] A. Beilinson and V. Drinfeld, Quantization of Hitchin Hamiltonians and Hecke Eigensheaves. Preprint, available at math.uchicago.edu/ mitya.

[BN4] D. Ben-Zvi and D. Nadler, The Character Theory of a Complex Group, arXiv:0904.1247.

[BFN] D. Ben-Zvi, J. Francis, and D. Nadler, Integral transforms and Drinfeld centers in derived algebraic geometry. arXiv:0805.0157 J. Amer. Math. Soc. 23 (2010), 909-966.

[BFN2] D. Ben-Zvi, J. Francis, and D. Nadler, Morita equivalence for convolution categories: Appendix to [BFN]. e-print arXiv:1209.0193.

[Be] D. Beraldo, Loop group actions on categories and Whittaker invariants. Berkeley PhD Thesis, 2013.

[DG] V. Drinfeld and D. Gaitsgory, On some finiteness questions for algebraic stacks. Geom. Funct. Anal. 23 (2013), no. 1, 149-294. e-print arXiv:1108.5351.

[G1] D. Gaitsgory, Generalities on DG categories. Available at http://www.math.harvard.edu/ gaitsgde/GL/

[G2] D. Gaitsgory, Sheaves of categories and the notion of 1-affineness. Stacks and categories in geometry, topology, and algebra, 127-225, Contemp. Math., 643, Amer. Math. Soc., Providence, RI, 2015. 
[GR] D. Gaitsgory and N. Rozenblyum, A study in derived algebraic geometry. Preliminary version (June 2016), available at http://www.math.harvard.edu/ gaitsgde/GL/

[Gi] V. Ginzburg, Admissible modules on a symmetric space. Orbites unipotentes et représentations, III. Astérisque No. 173-174 (1989), 9-10, 199-255.

[L1] J. Lurie, Higher topos theory. arXiv:math.CT/0608040 Annals of Mathematics Studies, 170. Princeton University Press, Princeton, NJ, 2009.

[L2] J. Lurie, Higher Algebra. Available at http://www.math.harvard.edu/ lurie/

[MV1] I. Mirković and K. Vilonen, Characteristic varieties of character sheaves. Invent. Math. 93 (1988), no. 2, $405-418$.

Department of Mathematics, University of Texas, Austin, TX 78712-0257

E-mail address: benzvi@math.utexas.edu

Department of Mathematics, University of Texas, Austin, TX 78712-0257

E-mail address: gunningham@math.utexas.edu 